\title{
The Signless Laplacian Spectral Radius of Unicyclic Graphs with Graph Constraints
}

\author{
Lihua FenG* AND Guihai Yu
}

School of Mathematics, Shandong Institute of Business and Technology 191 Binhaizhong Road, Yantai, Shandong, P.R. China, 264005

e-mail : fenglh@163.com

ABSTRACT. In this paper, we study the signless Laplacian spectral radius of unicyclic graphs with prescribed number of pendant vertices or independence number. We also characterize the extremal graphs completely.

\section{Introduction}

In this paper, we consider only simple connected graphs. Let $G$ be a simple graph with vertex set $V(G)$ and edge set $E(G)$. The adjacency matrix of $G$ is $A(G)=\left(a_{i j}\right)$ where $a_{i j}=1$ if two vertices $v_{i}$ and $v_{j}$ are adjacent in $G$ and 0 otherwise. Let $D(G)$ be the diagonal degree matrix of $G$. We call the matrix $L(G)=D(G)-A(G)$ the Laplacian matrix of $G$, while call the matrix $Q(G)=D(G)+A(G)$ the signless Laplacian matrix or $Q$-matrix of $G$. We denote the largest eigenvalues of $Q(G)$ by $\mu(G)$, and call it the signless Laplacian spectral radius (or the $Q$-spectral radius).

Let $K=K(G)$ be the vertex-edge incidence matrix of $G$. Thus $Q(G)=D(G)+$ $A(G)=K K^{t}$ and $K^{t} K=2 I_{m}+A\left(L_{G}\right)$, where $L_{G}$ is the line graph of $G$. Since $K K^{t}$ and $K^{t} K$ have the same nonzero eigenvalues, we can get that $\mu(G)=2+\rho\left(L_{G}\right)$. Since $Q(G)=K K^{t}$, we have that for any vector $x \in \mathrm{R}^{n}$, where $n$ is the order of $G, x^{t} Q(G) x=\sum_{u v \in E(G)}\left(x_{u}+x_{v}\right)^{2}$, where $x_{u}$ is the eigencomponent corresponding to the vertex $u$. So if $G$ is a connected graph, then $Q(G)$ is a symmetric, positive semidefinite and irreducible nonnegative matrix. By the Perron-Frobenius theorem, the largest eigenvalue of $Q(G)$ is a simple one and there is a unique (up to a factor) corresponding eigenvector known as Perron vector. Note that if we add edges to $G$, the spectral radius of $G$ will not decreases.

The unicyclic graph is a connected graph whose number of vertices equals to

\footnotetext{
* Corresponding author.
}

Received 7 December 2007; accepted 16 May 2008.

2000 Mathematics Subject Classification: 05C50.

Key words and phrases: unicyclic graph, independence number, signless Laplacian spectral radius.

This paper was supported Foundation of Education Committee of Shandong Province (J07YH03), Natural Science Foundation of Shandong Province(No.Y2006A17) and NSFSD(No.Y2006A17, Y2008A04). 
its number of edges. Let $G$ be a simple graph. A pendant vertex is a vertex of degree one. So for a unicyclic graph on $n$ vertices, it has at most $n-3$ pendant vertices. A subset $S$ of $V$ is called an independent set of $G$ if no two vertices in $S$ are adjacent in $G$. The independence number of $G$, denoted by $\alpha(G)$, is the size of a maximum independent set of $G$. It is easy to see that the independence number of a unicyclic graph on $n$ vertices is at most $n-2$. For two distinct vertices $u$ and $v$ of a connected graph $G$, the distance between $u$ and $v$, denoted by $d(u, v)$, is the length of a shortest path joining $u$ and $v$ in $G$. We use the standard notations in graph theory as in [12].

The study of the signless Laplacian spectral radius attracts researchers attention just recently. In [6], Fan et. al. studied the signless Laplacian spectral radius of bicyclic graph with fixed order. In [5], the authors discussed the smallest eigenvalue of $Q(G)$ as a parameter reflecting the nonbipartiteness of the graph $G$. Some other use of the signless Laplacian can be found in [1], [9], [3]. For a survey of this area, see [4]. For more results on spectral graph theory, we refer to [2].

We need the following graphs which would be helpful in the sequel. We use $\Delta_{n}^{k}$ to denote the unicyclic graph on $n$ vertices obtained from a cycle with three vertices $C_{3}$ by attaching $k$ paths of almost equal lengths at one vertex of $C_{3}$.

Let $K_{1, m+1}$ denote the star on $m+2$ vertices. If $\frac{n-1}{2} \leq m<n-1$, then $U_{n, m}^{*}$ is the unicyclic graph created from $K_{1, m+1}$ by first adding pendant edges to $n-m-2$ pendant vertices of $K_{1, m+1}$, then adding an edge among the rest of the pendant vertices of $K_{1, m+1}$, as shown in Fig. 1.

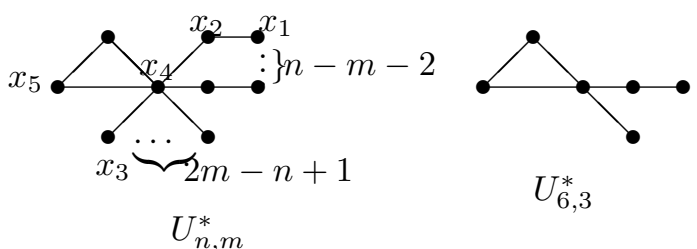

Fig. 1

For example, the graph $U_{6,3}^{*}$ is as shown in Fig. 1. Clearly, the graph $U_{n, m}^{*}$ has $n$ vertices, $m-1$ pendant vertices and independence number $m$.

For $\alpha \geq 3$. Let $C_{3}$ be the cycle with vertices $\left\{v_{1}, v_{2}, v_{3}\right\}$. The unicyclic graph $U_{n, \alpha}^{* *}$, as shown in Fig. 2, is obtained by first attaching one pendant edges to $v_{1}$ and $v_{2}$, respectively, and then attaching $2 \alpha-n+1$ pendant edges and $n-\alpha-3$ paths on two vertices at $v_{3}$. Clearly, $U_{n, \alpha}^{* *}$ has $n$ vertices, $\alpha$ pendant vertices and independence number $\alpha$. For example, $U_{6,3}^{* *}$ is shown in Fig. 2. 


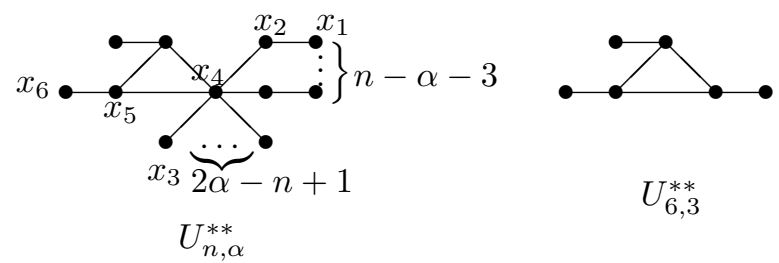

Fig. 2

In this paper, we study the signless Laplacian spectral radius of unicyclic graphs of order $n$ with prescribed number of pendant vertices or independence number, and determine the extremal graphs completely. Precisely, we get the following result.

Theorem 1.1. Let $G$ be a unicyclic graph on $n$ vertices with $k$ pendant vertices. Then $\mu(G) \leq \mu\left(\Delta_{n}^{k}\right)$, with equality if and only if $G=\Delta_{n}^{k}$.

Theorem 1.2. Let $G$ be a unicyclic graph on $n$ vertices with independence number $\alpha$. Then $\mu(G) \leq \mu\left(U_{n, \alpha}^{*}\right)$. The equality holds if and only if $G=U_{n, \alpha}^{*}$.

For convenience, we assume that the graph we considered in this paper has at least 3 vertices.

\section{Unicyclic graphs with $k$ pendant vertices}

Lemma 2.1. Let $G$ is a connected graph with maximum degree $\Delta$. Then $\Delta+1 \leq$ $\mu(G) \leq \max \left\{d_{u}+m_{u}\right\}$, where $m_{u}=\frac{\sum_{u v \in E(G)} d_{v}}{d_{u}}$. Moreover, the left equality holds if and only if $G$ is a star, and the right equality holds if and only if $G$ is regular or semiregular bipartite.

Proof. The left side can be found in [6]. For the right side, the proof is similar to that in [11], just consider the matrix $D+A$, and we omit the details.

Lemma 2.2([10]). Let $u, v$ be two vertices of the connected graph $G$ and $d_{v}$ be the degree of $v$, suppose $v_{1}, v_{2}, \cdots, v_{s} \in N(v) \backslash N(u)\left(1 \leq s \leq d_{v}\right)$, where $v_{1}, v_{2}, \cdots, v_{s}$ are different from $u$. Let $X=\left(x_{1}, x_{2}, \cdots, x_{n}\right)$ be the Perron vector of $Q(G)$, where $x_{i}$ corresponds to $v_{i},(1 \leq i \leq n)$. Let $H$ be the graph obtained from $G$ by deleting the edges $v v_{i}$ and adding the edges $u v_{i}, 1 \leq i \leq s$. If $x_{u} \geq x_{v}$, then $\mu(G)<\mu(H)$.

Lemma 2.3([8]). Let $u$ be a vertex of a connected graph $G$ with at least two vertices. Let $G(k, l), k>l \geq 1$, be the graph obtained from $G$ by attaching two paths $P_{k+1}=v_{1} v_{2} \cdots v_{k} u$ and $P_{l+1}=w_{1} w_{2} \cdots w_{l} u$ of length $k$ and $l$, respectively, at u. If $\Delta(G(k, l)) \geq 4$, then $\mu(G(k, l))<\mu(G(k-1, l+1))$.

Now, we consider the graph $G_{u v}$ obtained from the connected graph $G$ by subdividing the edge $u v$, that is, by replacing $u v$ with edges $u w, v w$, where $w$ is an additional vertex. We call the following two types of paths internal paths : (a) a 
sequence of vertices $v_{0}, v_{1}, \cdots, v_{k+1}(k \geq 2)$, where $v_{0}, v_{1}, \cdots, v_{k}$ are distinct, $v_{k+1}=$ $v_{0}$ of degree at least $3, d_{v_{i}}=2$ for $i=1, \cdots, k$, and $v_{i-1}$ and $v_{i}(i=1, \cdots, k+1)$ are adjacent. (b) A sequence of distinct vertices $v_{0}, v_{1}, \cdots, v_{k+1}(k \geq 0)$ such that $v_{i-1}$ and $v_{i}(i=1, \cdots, k+1)$ are adjacent, $d_{v_{0}} \geq 3, d_{v_{k+1}} \geq 3$ and $d_{v_{i}}=2$ whenever $1 \leq i \leq k$.

Lemma 2.4([7]). Let $G$ be a connected graph and uv be some edge on the internal path of $G$ as we defined above. If we subdivide uv, that is, substitute it by uw, wv, with the new vertex $w$, and denote the new graph by $G_{u v}$, then $\mu\left(G_{u v}\right)<\mu(G)$.

Now, we can present the proof of Theorem 1.1.

Proof of Theorem 1.1. Let $G$ be a unicyclic graph of order $n$ with $k$ pendant vertices and maximal signless Laplacian spectral radius. Let $X$ be the eigenvector corresponding to $\mu=\mu(G)$, and suppose the eigencomponent corresponding to the vertex $v$ is $x_{v}$. Further, let $C$ be the unique cycle of $G$ and $u_{1}, u_{2}, \cdots, u_{t}, t \geq 1$ be the vertices on $C$ having degree at least 3 . Suppose the trees attached to $C$ are rooted at $u_{i}$. We discuss in two cases.

(1) If $t=1$, then there is only one vertex $u_{1}$ on $C$ having degree 3 . If there are vertices of degree at least three outside $C$. Suppose $w$ is such a vertex that has minimal distance from $u$. If the distance from $u$ and $w$ at least 1 , then by Lemma 2.4, contract the internal path between $u$ and $w$, the signless Laplacian spectral radius does not decrease. Hence there are no vertices of degree at least three outside $C$ in this case. If the length of the cycle $C$ is greater than 3 , then by Lemma 2.4, we can contracting the internal path on $C$ to make $C$ be a triangle $C_{3}$, then subdividing the pendant path outside $C$, the signless Laplacian spectral radius increases. Hence, the length of the cycle $C$ is 3 . If the lengths of the pendant paths rooted at $u_{1}$ are not almost equal, by using Lemma 2.3 , we can get the result.

(2) If $t \geq 2$. By Lemma 2.2, comparing the eigencomponents of $u_{1}, u_{2}, \cdots, u_{t}$, we can get a new graph with larger signless Laplacian spectral radius. So this is impossible. If the length of $C$ is greater than 4 , then by Lemma 2.4, we can contract the internal path on $C$ to make $C$ a triangle, in this way, the signless Laplacian spectral radius does not decrease. So this is also impossible.

Corollary 2.5. Let $1 \leq k<n-3$. Then $\mu\left(\Delta_{n}^{k}\right)<\mu\left(\Delta_{n}^{k+1}\right)$.

Proof. Since $k<n-3$, it follows that there is pendant path $P_{l}=v_{1} v_{2} \cdots v_{l}$ attached to the root vertex $u$ of $\Delta_{n}^{k}$ such that $l \geq 2$. Let $G=\Delta_{n}^{k}-\left\{v_{l-1} v_{l}\right\}+\left\{u v_{l}\right\}$. Obviously, $G$ is a unicyclic graph with $k+1$ pendant vertices. By Lemma 2.3, we have $\mu\left(\Delta_{n}^{k}\right)<\mu(G)$, by Theorem 1.1 , we have $\mu(G)<\mu\left(\Delta_{n}^{k+1}\right)$. Hence we get the result.

Corollary 2.6. Of all unicyclic graphs on $n$ vertices, $S_{n}^{*}$ has the maximum signless Laplacian spectral radius, where $S_{n}^{*}$ is obtained from the star on $n$ vertices $S_{n}$ by joining any two vertices of degree one. 


\section{Unicyclic graph with independence number $\alpha$}

\subsection{Useful lemmas}

The next lemma plays an important role in our paper. We use the notations in [12]: $\alpha$ is the vertex independence number. $\alpha^{\prime}$ is the edge independence number or matching number. $\beta$ is the vertex covering number. $\beta^{\prime}$ is the edge covering number.

The following well known relation is called König-Egerváry theorem: $\alpha+\beta=$ $\alpha^{\prime}+\beta^{\prime}=n$.

Lemma 3.1. Let $G$ be a non-bipartite unicyclic graph with $n$ vertices and independence number $\alpha(G)$. Suppose the unique cycle is $C$, then $\alpha(G) \geq\left\lceil\frac{n}{2}\right\rceil-1$, with equality if and only if $G-V(C)$ has a perfect matching.

Proof. The cycle $C$ must have odd length, say $k$. Let $e$ be an edge of $C$. The graph $G-e$ is bipartite, so $\alpha(G-e) \geq\left\lceil\frac{n}{2}\right\rceil$. An independent set $S$ in $G-e$ is also independent in $G$ unless it contains both endpoints of $e$. If $|S|>\left\lceil\frac{n}{2}\right\rceil$, then we can afford to drop one of these vertices. If $|S|=\left\lceil\frac{n}{2}\right\rceil$, then we can take the other partite set instead to avoid the endpoints of $e$. In each case, $\alpha(G) \geq\left\lfloor\frac{n-1}{2}\right\rfloor=\left\lceil\frac{n}{2}\right\rceil-1$. If $G-V(C)$ has a perfect matching, then an independent set is limited to $\frac{k-1}{2}$ vertices of $C$ and $\frac{n-k}{2}$ vertices outside $C$, so $\alpha(G) \leq \frac{n-1}{2}$ and equality holds.

For the converse, observe that deleting $E(C)$ leaves a forest $F$ in which each component has a vertex of $C$. Let $H$ be a component of $F$, with $u$ being its vertex on $C$, and let $r$ be its order. If $H-u$ has no perfect matching, then $\alpha^{\prime}(H-u) \leq\left\lfloor\frac{r}{2}\right\rfloor-1$ (that is, it cannot equal $\frac{r-1}{2}$ ). Now $\beta(H-u) \leq\left\lfloor\frac{r}{2}\right\rfloor-1$ by König-Egerváry theorem, and $\alpha(H-u) \geq\left\lceil\frac{r}{2}\right\rceil$, since the complement of a vertex cover is an independent set. Since this independent set does not use $u$, we can combine it with an independent set of size at least $\left\lceil\frac{n-r}{2}\right\rceil$ in the bipartite graph $G-V(H)$ to obtain $\alpha(G) \geq\left\lceil\frac{n}{2}\right\rceil$. Since this holds for each component of $F, \alpha(G)=\left\lfloor\frac{n-1}{2}\right\rfloor$ requires a perfect matching in $G-V(C)$.

Lemma 3.2. Let $G$ be a unicyclic graph with $n$ vertices and independence number $\alpha(G)$. Then $\alpha(G) \geq \frac{n-1}{2}$.

Proof. If $G$ is bipartite, the $\alpha(G) \geq\left\lceil\frac{n}{2}\right\rceil \geq \frac{n-1}{2}$. If $G$ is non-bipartite, then by Lemma 3.1, $\alpha(G) \geq\left\lceil\frac{n}{2}\right\rceil-1$. If $n$ is odd, then $\left\lceil\frac{n}{2}\right\rceil-1=\frac{n-1}{2}$. If $n$ is even, 
suppose the unique cycle is $C$, then the equality in Lemma 3.1 would not happen, since $G$ contains an odd cycle and $G-V(C)$ has odd number of vertices. Hence in this case, we also have $\alpha(G) \geq\left\lceil\frac{n}{2}\right\rceil \geq \frac{n-1}{2}$.

Remark. If $m \geq \frac{n-1}{2}$, then $\Delta_{n}^{m-1}$ is identical to $U_{n, m}^{*}$.

Lemma 3.3. Let $G$ be a unicyclic graph with $n \geq 3$ vertices and independence number $\alpha(G)$. Then $G$ has at most $\alpha(G)$ pendant vertices.

Proof. This is since all the pendant vertices form an independent set of $G$.

\subsection{Main results}

If $\alpha=1$, the unique unicyclic graph is $C_{3}=U_{3,1}^{*}$.

Theorem 3.4. Let $G$ be a unicyclic graphs of order $n \geq 3$ with $p$ pendant vertices and independence number $\alpha \geq 2$. If $p \leq \alpha-1$, then $\mu(G) \leq \mu\left(U_{n, \alpha}^{*}\right)$, with equality holding if and only if $G=U_{n, \alpha}^{*}$.

Proof. Let $G$ be a unicyclic graph with $n \geq 3$ vertices and independence number $\alpha(G)$. Suppose that $G$ has $p$ pendant vertices. By Theorem 1.1, we have $\mu(G) \leq \mu\left(\Delta_{n}^{p}\right)$.

Now, by Lemmas 3.2, 3.3 and Corollary 2.5, we have $\mu\left(\Delta_{n}^{p}\right) \leq \mu\left(\Delta_{n}^{\alpha-1}\right)=\mu\left(U_{n, \alpha}^{*}\right)$, since $\Delta_{n}^{\alpha-1}=U_{n, \alpha}^{*}$ for $\alpha(G) \geq \frac{n-1}{2}$.

Moreover, the first equality holds if and only if $G$ is uniquely at $\Delta_{n}^{p}$ and the second equality holds if and only if $p=\alpha-1$. Hence we complete the proof.

Next, we consider the case when the number $p$ of pendant vertices of a unicyclic graph is equal to its independence number $\alpha$.

Let $G$ be a unicyclic graph and $C$ be the cycle of $G$ with $V(C)=\left\{v_{1}, v_{2}, \cdots, v_{t}\right\}$, $t \geq 3$. Note that $p=\alpha$, then each $v_{i}(1 \leq i \leq t)$ has at least one pendant vertex as its neighbor, since otherwise this would increase the independence number of $G$. So we have $t \leq n-\alpha$. Since $t \geq 3$, we have $n \geq \alpha+3$.

If $t$ is even, then $G$ is bipartite, and $\alpha(G) \geq\left\lceil\frac{n}{2}\right\rceil \geq \frac{n}{2}$. If $t$ is odd, then $G$ is non-bipartite. By Lemma 3.1, the equality in Lemma 3.1 would not happen, since $G$ contains an odd cycle and $G-V(C)$ has odd number of vertices. Hence in this case, we also have $\alpha(G) \geq\left\lceil\frac{n}{2}\right\rceil \geq \frac{n}{2}$. Hence in either case, we have $n \leq 2 \alpha$.

If $\alpha=1$ or 2 , there does not exist unicyclic graphs such that $p=\alpha$.

In the following, we shall assume that $\alpha \geq 3$.

If $\alpha=3$, then $n \leq 2 \alpha=6$. The unicyclic graph with at most 6 vertices satisfying $p=\alpha=3$ is uniquely $U_{6,3}^{* *}$, where $U_{6,3}^{* *}$ is shown in Fig.2.

If $G$ has $p=\alpha \geq 4$ pendant vertices, then using Lemma 2.2 on vertices of $V(C)=$ $\left\{v_{1}, v_{2}, \cdots, v_{t}\right\}$, and by Lemma 2.4 if there are internal paths in the trees attached, and adding pendant edges to the pendant vertices if necessary, we have $\mu(G) \leq$ 
$\mu\left(U_{n, \alpha}^{* *}\right)$. Hence we have the following result.

Theorem 3.5. Let $G$ be a unicyclic graphs of order $n \geq 6$ with $p$ pendant vertices and independence number $\alpha \geq 3$. If $p=\alpha$, then $\mu(G) \leq \mu\left(U_{n, \alpha}^{* *}\right)$, with equality holding if and only if $G=U_{n, \alpha}^{* *}$.

Theorem 3.6. For $3 \leq \alpha \leq n-3$, we have $\mu\left(U_{n, \alpha}^{*}\right)>\mu\left(U_{n, \alpha}^{* *}\right)$.

Proof. Suppose $4 \leq \alpha \leq n-3$. Then by Lemma 2.1, $\mu\left(U_{n, \alpha}^{*}\right) \geq 1+\Delta=2+\alpha \geq 6$. For $U_{n, \alpha}^{*}$, let $X$ be its Perron vector. By symmetry, suppose the eigencomponents of $X$ as shown in Figure 1. Then from $\mu X=(D+A) X$, we have,

$$
\begin{aligned}
\mu x_{1} & =x_{1}+x_{2}, \\
\mu x_{2} & =2 x_{2}+x_{1}+x_{4}, \\
\mu x_{3} & =x_{3}+x_{4}, \\
\mu x_{4} & =(\alpha+1) x_{4}+2 x_{5}+(2 \alpha-n+1) x_{3}+(n-\alpha-2) x_{2}, \\
\mu x_{5} & =2 x_{5}+x_{5}+x_{4} .
\end{aligned}
$$

Simplifying the above equation, $\mu$ satisfies the equation

$$
\mu-\alpha-1=\frac{2}{\mu-3}+\frac{2 \alpha-n+1}{\mu-1}+\frac{n-\alpha-2}{\mu-2-\frac{1}{\mu-1}} .
$$

Similarly, for $U_{n, \alpha}^{* *}$, by symmetry, we can suppose the eigencomponents are as shown in Figure 2. From $\mu X=(D+A) X$, we have

$$
\begin{aligned}
& \mu x_{1}=x_{1}+x_{2}, \\
& \mu x_{2}=2 x_{2}+x_{1}+x_{4}, \\
& \mu x_{3}=x_{3}+x_{4}, \\
& \mu x_{4}=\alpha x_{4}+2 x_{5}+(2 \alpha-n+1) x_{3}+(n-\alpha-3) x_{2}, \\
& \mu x_{5}=3 x_{5}+x_{5}+x_{4}+x_{6}, \\
& \mu x_{6}=x_{5}+x_{6} .
\end{aligned}
$$

Simplifying the above equation, $\mu$ satisfies the equation

$$
\mu-\alpha=\frac{2}{\mu-4-\frac{1}{\mu-1}}+\frac{2 \alpha-n+1}{\mu-1}+\frac{n-\alpha-3}{\mu-2-\frac{1}{\mu-1}} .
$$

From equation (1), we have

$$
\frac{2 \alpha-n+1}{\mu-1}=\mu-\alpha-1-\frac{2}{\mu-3}-\frac{n-\alpha-2}{\mu-2-\frac{1}{\mu-1}} .
$$

Let

$$
f(\mu)=\mu-\alpha-\frac{2}{\mu-4-\frac{1}{\mu-1}}-\frac{2 \alpha-n+1}{\mu-1}-\frac{n-\alpha-3}{\mu-2-\frac{1}{\mu-1}} .
$$


Take (3) into $f(\mu)$, we have

$$
\begin{aligned}
f(\mu) & =1+\frac{2}{\mu-3}+\frac{1}{\mu-2-\frac{1}{\mu-1}}-\frac{1}{\mu-4-\frac{1}{\mu-1}} \\
& =\frac{\mu-6-\frac{1}{\mu-1}}{\mu-4-\frac{1}{\mu-1}}+\frac{2}{\mu-3}+\frac{1}{\mu-2-\frac{1}{\mu-1}} \\
& =\frac{\mu-6}{\mu-4-\frac{1}{\mu-1}}-\frac{1}{\mu^{2}-5 \mu+3}+\frac{2}{\mu-3}+\frac{1}{\mu-2-\frac{1}{\mu-1}} .
\end{aligned}
$$

Since $\mu\left(U_{n, \alpha}^{*}\right) \geq 6$, we have, if $\mu=\mu\left(U_{n, \alpha}^{*}\right)$, then $-\frac{1}{\mu^{2}-5 \mu+3}+\frac{2}{\mu-3}>0$, and $f\left(\mu\left(U_{n, \alpha}^{*}\right)\right)>0$, so we have $\mu\left(U_{n, \alpha}^{*}\right)>\mu\left(U_{n, \alpha}^{* *}\right)$.

If $\alpha=3$, by using a similar method, we also have $\mu\left(U_{6,3}^{* *}\right)<\mu\left(U_{6,3}^{*}\right)$.

So we complete the proof.

Now, we can present the proof of Theorem 1.2.

Proof of Theorem 1.2. Suppose $G$ has $p$ pendant vertices. By Lemma 3.2, we have $\frac{n-1}{2} \leq \alpha \leq n-2$. We discuss in the following cases.

(a) If $\alpha=1$, then $n=3$, and the unique unicyclic graph is $U_{3,1}^{*}=C_{3}$.

(b) If $\alpha=2$, then $4 \leq n \leq 5$. If $p=1$ and $n=4,5$, then $\mu(G) \leq \mu\left(U_{n, 2}^{*}\right)$, with equality holding if and only if $G=U_{n, 2}^{*}$. If $p=2$, there does not exist such unicyclic graph.

(c) If $\alpha=3$, then $5 \leq n \leq 7$. If $p \leq 2$, then by Theorem $3.4, \mu(G) \leq \mu\left(U_{n, 3}^{*}\right)$, with equality holding if and only if $G=U_{n, 3}^{*}$. If $p=3$, the unique unicyclic graph is $U_{6,3}^{*}$. By Theorem 3.6, we have $\mu(G) \leq \mu\left(U_{6,3}^{*}\right)$, with equality holding if and only if $G=U_{6,3}^{*}$.

(d) If $4 \leq \alpha \leq n-3$, then by Theorems $3.4,3.5,3.6$, we have $\mu(G) \leq \mu\left(U_{n, \alpha}^{*}\right)$, with equality holding if and only if $G=U_{n, \alpha}^{*}$.

(e) If $\alpha=n-2$, note $p \leq n-3$, then by Theorem 3.4, we have $\mu(G) \leq \mu\left(U_{n, \alpha}^{*}\right)$, with equality holding if and only if $G=U_{n, \alpha}^{*}$.

Combining the above discussion, we get the result.

At last, we estimate the signless Laplacian spectral radius of unicyclic graph described above.

Theorem 3.7. The signless Laplacian spectral radius of $U_{n, \alpha}^{*}$ satisfies $\alpha+2<$ $\mu\left(\left(U_{n, \alpha}^{*}\right) \leq \alpha+3\right.$. The right equality holds if and only if $\alpha=1, n=3$, i.e., the graph is $U_{3,1}^{*}=C_{3}$.

Proof. From Lemma 2.1, we can get the result directly.

Acknowledgment. The authors are grateful to Professor Li Qiao (in Shanghai Jiaotong University) for his help. 


\section{References}

[1] R. B. Bapat, J. W. Grossman, D. M. Kulkarni, Generalized matrix tree theorem for mixed graphs, Linear and Multilinear Algebra, 46(4)(1999), 299-312.

[2] D. Cvetković, M. Doob, H. Sachs, Spectra of Graphs, Academic Press, New York, 1980.

[3] D. Cvetković, Signless Laplacians and line graphs, Bull. Acad. Serbe Sci. Ars. Cl. Sci. Math. Nat. Sci. Math., 131(30) (2005), 85-92.

[4] D. Cvetković, P. Rowlinson, S. K. Simić, Signless Laplacians of finite graphs, Linear Algebra Appl., 423(2007), 155-171.

[5] M. Desai, V. Rao, A characterizaion of the smallest eigenvalue of a graph, J. Graph Theory, 18(1994), 181-194.

[6] Y. Fan, B. S. Tam, J. Zhou, Maximizing spectral radius of unoriented Laplacian matrix over bicyclic graphs of a given order, Linear Multilinear Algebra, 56(2008), 381-397.

[7] L. Feng, Q. Li, X. D. Zhang, Minimizing the Laplacian spectral radius of trees with given matching number, Linear Multilinear Algebra, 55(2)(2007), 199-207.

[8] L. Feng, G. Yu, No starlike trees are Laplacian cospectral, Univ. Beograd, Publ. Elektrotehn. Fak., Ser. Mat, 18(2007), 46-5.

[9] J. W. Grossman, D. M. Kulkarni, I.Schochetman, Algebraic graph theory without orientation, Linear Algebra Appl., 212/213(1994), 289-307.

[10] Y. Hong, X. D. Zhang, Sharp upper and lower bounds for largest eigenvalue of the Laplacian matrices of trees, Discrete Math., 296(2005), 187-197.

[11] R. Merris, A note on Laplacian graph eigenvalues, Linear Algebra Appl., 285(13)(1998), 33-35.

[12] D. B. West, Introduction to Graph Theory, Prentice -Hall, 2001. 